\title{
Case for diagnosis. Metastatic Crohn's disease ${ }^{*}$
}

\author{
João Renato Vianna Gontijo ${ }^{1}$ \\ Maria Silvia Laborne Alves de Sousa ${ }^{1,2}$
}

\author{
Franciele Antonieta Bianchi Leidenz ${ }^{1}$
}

DOI: http:/ / dx.doi.org/10.1590/abd1806-4841.20165731

\section{CASE REPORT}

A 14-year-old girl, with weight and height appropriate for her age, presented with pruritic, erythematous papules and plaques on the anterior and posterior trunk that had been present for two months (Figures 1 and 2). At 11 years of age, the patient was diagnosed with severe Crohn's disease (CD), refractory to multiples treatments. At the time of her first appointment in our clinic, she was using infliximab, $10 \mathrm{mg} / \mathrm{kg}$, with a persistent perianal ulcer and chronic diarrhea, and levothyroxine for controlled hypothyroidism. The patient denied contact with contaminated water or ponds. A skin biopsy from her back showed superficial and deep perivascular lymphohistiocytic inflammatory infiltrate, associated with perifolliculitis and formation of poorly defined granulomas (Figure 3). Culture and special stains for bacteria, fungi, and parasites were negative. As diarrhea worsened, a course of $60 \mathrm{mg} /$ day of prednisone was prescribed, resulting in a significant improvement of the intestinal condition and total resolution of skin lesions (Figure 4).

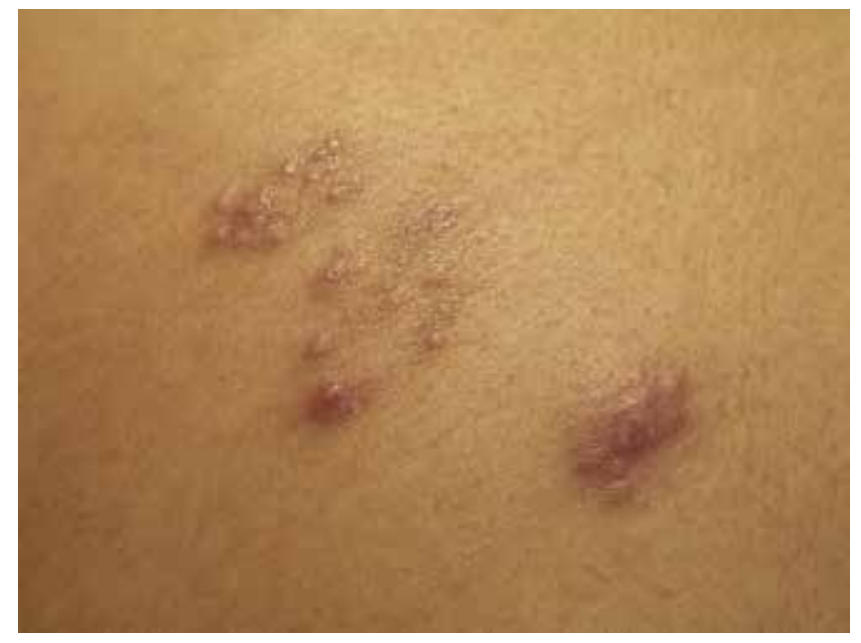

FIGURE 1: Multiple erythematous papules, isolated or in plaques, on the back 


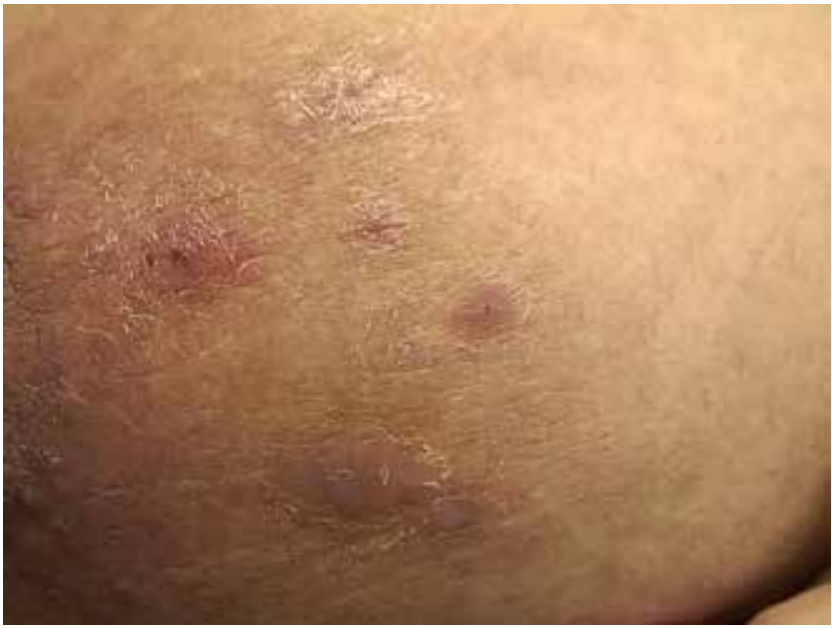

Figure 2: Erythematous scaly papules on the anterior trunk

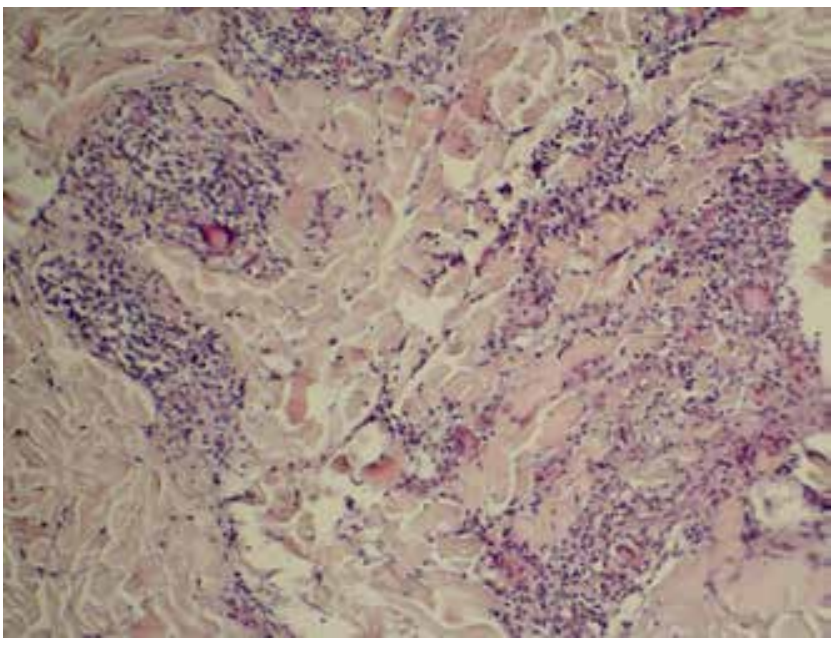

FIGURE 3: Superficial perivascular lymphocytic infiltrate with poorly defined granuloma

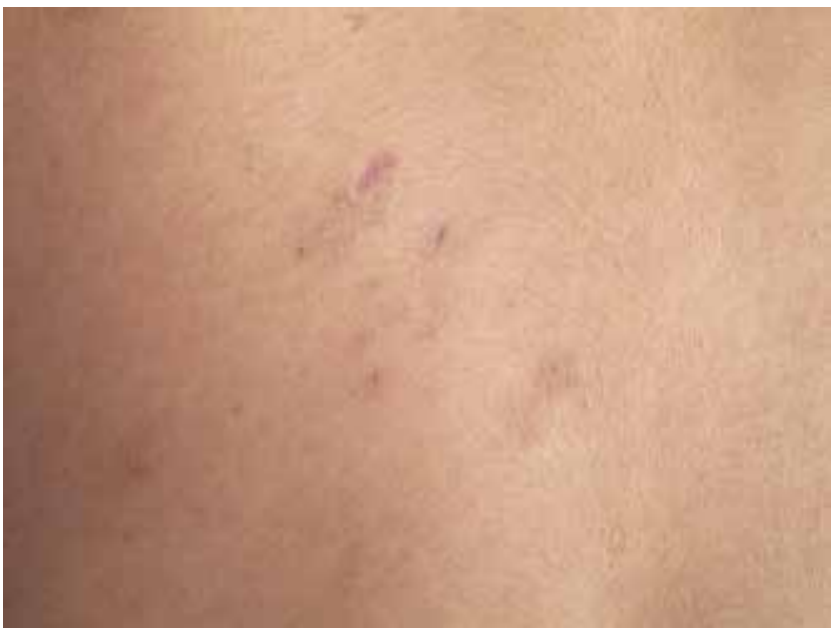

Figure 4: Resolution of lesions and scar on the biopsy site

\section{DISCUSSION}

Metastatic Crohn's disease (MCD) is a rare cutaneous manifestation, defined by the presence of non-caseous granulomatous skin lesions that are discontinuous to the affected gastrointestinal tract in Crohn's disease (CD) patients. The pathogenesis is poorly understood, and it is unlikely that it represents a dissemination of the bowel disease. ${ }^{1}$ Some authors believe that the distant granulomatous reaction may be caused by the deposition of immunocomplexes, or by type IV hypersensitivity reaction..$^{1,2}$

Up to $44 \%$ of patients with CD have skin manifestations. Perianal fistulas and fissures are the most frequent presentation and MCD the rarest skin finding. ${ }^{2,3}$ MCD mostly affects young adults, and its prevalence and incidence are likely to be underestimated. To date, only about 100 cases have been reported. ${ }^{4}$

The dermatological findings are nonspecific and heterogeneous. In children, the most frequent presentation is genital edema. ${ }^{1}$ In adults, the presence of nodules and/or plaques is more common, especially in the lower limbs. ${ }^{1}$ There is no established correlation between the activity of the skin lesions and gastrointestinal impairment. In most cases, MCD affects patients with ongoing CD; however, it may precede the gastrointestinal condition, making diagnosis even more difficult., ${ }^{5,6}$

Other skin manifestations associated with $\mathrm{CD}$ are erythema nodosum, oral aphthae, pyoderma gangrenosum, as well as autoimmunities and dermatoses secondary to nutritional deficiency. ${ }^{7}$

Cutaneous histological findings are non-caseous granulomas, with Langerhans and foreign body giant cells, histiocytes, lymphocytes, plasmocytes, and eosinophils, which are similar to gastrointestinal findings. Necrobiosis and perivascular granulomatosis have also been described. ${ }^{5,6}$

Since cutaneous lesions are clinically inespecific, the diagnosis of MCD relies on the dermopathological findings and on the exclusion of other granulomatous diseases and infections. ${ }^{6}$ The main differential diagnosis is sarcoidosis; however, in our country, ectopic schistosomiasis, fungal and mycobacterial infections should also be excluded.

Treatment is based on case reports and is usually unsatisfactory. Topical and oral steroids are the mainstay of treatment, followed by metronidazole. Severe and refractory cases usually respond to TNF alpha inhibitors, particularly infliximab. Although the patient was being treated with the latter, cutaneous lesions showed significant improvement with the introduction of oral steroids. The use of other immunosuppressant's drugs, thalidomide, surgery, and topic tacrolimus has also been reported. ${ }^{2,8} \square$ 
Abstract: Metastatic Crohn's disease is a rare skin manifestation, defined by granulomatous skin lesions that are discontinuous to the affected gastrointestinal tract and histopathologically resembling inflammatory bowel lesions. Up to $44 \%$ of patients with Crohn's disease have cutaneous manifestations, of which metastatic lesions are the least common. We present a case of an adolescent with refractory Crohn's disease and persistent papules and plaques on the skin.

Keywords: Crohn's disease; Granuloma; Granulomatous disease, chronic; Skin manifestations

\section{REFERENCES}

1. Palamaras I, El-Jabbour J, Pietropaolo N, Thomson P, Mann S, Robles W, et al. Metastatic Crohn's disease: a review. J Eur Acad Dermatol Venereol. 2008;22:1033-43.

2. Kurtzman DJ, Jones T, Lian F, Peng LS. Metastatic Crohn's disease: A review and approach to therapy. J Am Acad Dermatol. 2014;71:804-13.

3. Keiler S, Tyson P, Tamburro J.. Metastatic cutaneous Crohn's disease in children: Case report and review of literature. Pediatr Dermatol. 2009;26:604-9.

4. Lang N, Hartschuh W, Enk A, Toberer F. Metastatic Crohn's disease: a diagnostic and therapeutic challenge. J Dtsch Dermatol Ges. 2015;13:571-4.

5. Hackzell-Bradley M, Hedblad MA, Stephansson EA. Metastatic Crohn's disease. Report of 3 cases with special reference to histopathologic findings. Arch Dermatol. 1996;132:928-32.

6. Emanuel PO, Phelps RG. Metastatic Crohn's disease: a histopathologic study of 12 cases. J Cutan Pathol. 2008;35:457-61.

7. Burgdorf W. Cutaneous manifestations of Crohn's disease. J Am Acad Dermatol. 1981;5:689-95.

8. Guest GD, Fink RL. Metastatic Crohn's disease. Case report of na unusual variant and review of the literature. Dis Colon Rectum. 2000;43:1764-6.
MAILING ADDRESS:

João Renato Vianna Gontijo

Av. Francisco Sales, 1111

Santa Efigênia

30150-221 - Belo Horizonte - MG

Brazil

E-mail: joaorenato20@hotmail.com

How to cite this article: Gontijo JRV, Leidenz FAB, Sousa MSLA. Case for diagnosis. Metastatic Crohn's disease. An Bras Dermatol. 2016;91(4):531-3. 